\title{
Relationship between high-frequency activity in the sensory and the motor hand areas, and regional myelin content
}

Leo Tomasevic ${ }^{1 *}$, Hartwig Roman Siebner ${ }^{1,2,3}$, Axel Thielscher ${ }^{1,4}$, Fiore Manganelli ${ }^{5}$, Giuseppe Pontillo ${ }^{6}$, Raffaele Dubbioso ${ }^{5 *}$.

${ }^{1}$ Danish Research Centre for Magnetic Resonance, Center for Functional and Diagnostic Imaging and Research, Copenhagen University Hospital Amager and Hvidovre, Copenhagen, Denmark.

${ }^{2}$ Department of Neurology, Copenhagen University Hospital Bispebjerg, Copenhagen, Denmark.

${ }^{3}$ Institute for Clinical Medicine, Faculty of Health and Medical Sciences, University of Copenhagen, Copenhagen, Denmark

${ }^{4}$ Department of Health Technology, Technical University of Denmark, Kgs. Lyngby, Denmark.

${ }^{5}$ Department of Neurosciences, Reproductive Sciences and Odontostomatology, University Federico II of Naples, Naples, Italy.

${ }^{6}$ Department of Advanced Biomedical Sciences, University Federico II of Naples, Naples, Italy

Word count: Abstract: 261; Main text: 3750; Number of figures: 3.

\section{*Corresponding authors:}

Leo Tomasevic, PhD

Danish Research Centre for Magnetic Resonance (DRCMR), Center for Functional and Diagnostic Imaging and Research, Copenhagen University Hospital Hvidovre, Kettegård Allé 30, DK-2650 Hvidovre, Denmark. Phone: +45 3862 6678, e-mail: leot@drcmr.dk

Raffaele Dubbioso, MD, PhD

Department of Neurosciences, Reproductive Sciences and Odontostomatology, University Federico II of Napoli,

Via Sergio Pansini, 5. 80131 Napoli, Italy.

Phone: +39 0817464579, e-mail: rafdubbioso@gmail.com 


\section{CRediT authorship contribution statement}

Raffaele Dubbioso: Conducting the experimental work/data collection/obtaining access to data, Conducting the analysis of data, Interpretation of the results, Writing of the first draft of the manuscript, Finalisation of the manuscript and submission.

Fiore Manganelli: Interpretation of the results, Writing of the first draft of the manuscript.

Giuseppe Pontillo: Conducting the experimental work/data collection/obtaining access to data, Writing of the first draft of the manuscript.

Axel Thielscher: Development of the key methods, Interpretation of the results.

Hartwig Roman Siebner: Interpretation of the results, Writing of the first draft of the manuscript, Finalisation of the manuscript and submission.

Leo Tomasevic: Planning of the experiments and methodology design and development, Interpretation of the results, Development of the key methods, Writing of the first draft of the manuscript, Finalisation of the manuscript and submission.

\section{Conflict of Interest}

Hartwig R. Siebner has received honoraria as speaker from Sanofi Genzyme, Denmark and Novartis, Denmark, as consultant from Sanofi Genzyme, Denmark, Lophora, Denmark, and Lundbeck AS, Denmark, and as editorin-chief (Neuroimage Clinical) and senior editor (NeuroImage) from Elsevier Publishers, Amsterdam, The Netherlands. He has received royalties as book editor from Springer Publishers, Stuttgart, Germany and from Gyldendal Publishers, Copenhagen, Denmark. The other authors report no conflict of interests.

\section{Funding}

Leo Tomasevic received support from the Lundbeck Foundation (Experiment grant "Brain mapping of GABAergic inhibition”, R346-2020-1822).

Hartwig R. Siebner received support from the Lundbeck Foundation (Collaborative grant "ADAptive and Precise Targeting of cortex-basal ganglia circuits in Parkinson’s Disease”, R336-2020-1035) and holds a 5-year professorship in precision medicine at the Faculty of Health Sciences and Medicine, University of Copenhagen which is sponsored by the Lundbeck Foundation (Grant Nr. R186-2015-2138).

Axel Thielscher received support from the Lundbeck Foundation (grant R313-2019-622). 


\begin{abstract}
Background: The human primary sensory (S1) and primary motor (M1) hand areas feature high-frequency neuronal responses. Electrical nerve stimulation evokes high-frequency oscillations (HFO) at around $650 \mathrm{~Hz}$ in the contralateral S1. Likewise, paired-pulse transcranial magnetic stimulation of M1 produces short interval intracortical facilitation (SICF) of motor evoked potentials in contralateral hand muscles. SICF features several peaks of facilitation which are separated by inter-peak intervals resembling HFO rhythmicity.
\end{abstract}

Hypothesis: In this study, we tested the hypothesis that the individual expressions of HFO and SICF are tightly related to each other and to the regional myelin content in the sensorimotor cortex.

Methods: In 24 healthy volunteers, we recorded HFO and SICF, and, in a subgroup of 20 participants, we mapped the cortical myelin content using the ratio between the T1- and T2-weighted MRI signal as read-out.

Results: The individual frequencies and magnitudes of HFO and SICF were tightly correlated: the intervals between the first and second peak of cortical HFO and SICF showed a positive linear relationship (r=0.703, $\mathrm{p}<$ 0.001), while their amplitudes were inversely related $(r=-0.613, p=0.001)$. The rhythmicity, but not the magnitude of the high-frequency responses, was related to the cortical myelin content: the higher the cortical myelin content, the shorter the inter-peak intervals of HFO and SICF.

Conclusion: The results confirm a tight functional relationship between high-frequency responses in S1 (i.e., HFO) and M1 (i.e., SICF). They also establish a link between the degree of regional cortical myelination and the expression of high-frequency responses in the human cortex, giving further the opportunity to infer their possible generators.

Keywords: High-frequency oscillation; transcranial magnetic stimulation; cortical myelin content; magnetic resonance imaging; primary somatosensory cortex, primary motor cortex.

Abbreviations: AMT = Active Motor Threshold; M1= Primary Motor Cortex; HFO = High Frequency Oscillations; RMT= Resting Motor Threshold; S1= Primary Somatosensory Cortex; SICF = Short Interval Intracortical Facilitation; SEP = Somatosensory Evoked Potential; TMS = Transcranial Magnetic Stimulation. 


\section{Introduction}

Peripheral sensory stimulation produces a complex set of regional responses in the contralateral somatosensory cortex, where afferent input is processed, and that can be detected by electroencephalography (EEG). For instance, electrical nerve stimulation at the wrist does not only evoke an early somatosensory evoked potential (SEP) at around $20 \mathrm{~ms}$ (N20) in the contralateral primary somatosensory area (S1) [1] but concurrently induces high-frequency oscillations (HFO) at about $650 \mathrm{~Hz}$ [1-5]. HFO are composed of two independent bursts, probably the first of subcortical (early-HFO) and the second of cortical origin (late-HFO), arising respectively before and after N20 [4,6]. Late-HFO are generated in S1, namely in Brodmann areas 3B (BA3B)[2] and 1 (BA1) [2], and are associated respectively to peaks from 1 to 3, and to peaks from 3 to 5 in the burst following N20 [7]. A study in monkeys employed concurrent extracellular single-unit recordings to show that the activity in BA3 is generated by bursts of 2 to 5 action potentials likely arising from pyramidal neurons, as suggested by the duration of the action potentials and their detectability with the EEG $[8,9]$.

Phenomena that feature a rhythmic pattern at around $650 \mathrm{~Hz}$ can also be elicited in the primary motor area (M1) using paired-pulse transcranial magnetic stimulation (TMS). If two TMS pulses are applied at interstimulus intervals ranging from 0.6 to 5 milliseconds and at an intensity around cortical motor threshold [10], or a subthreshold pulse after a suprathreshold one [11], it is possible to observe several peaks of facilitation of the motor evoked potentials (MEPs) at specific inter-stimulus intervals. The rhythmicity of these peaks of short interval intracortical facilitation (SICF) closely resembles the one of sensory evoked HFO in S1 or the rhythmicity of the descending I-waves evoked by TMS in the corticospinal tract [11-14]. While its exact neural origin is still debated, SICF is thought to reflect the strength and temporal dynamics of cortical circuits that facilitate transsynaptic excitation of those pyramidal cells in M1 that send direct monosynaptic projections to the spinal motoneurons $[11,14,15]$.

Given the cortical origin and shared rhythmicity of late HFO and SICF, it has been hypothesized that these phenomena may be closely related $[8,16]$. Yet, studies, that have directly examined whether and how the individual expression of HFO and SICF are related to each other, are lacking. Another question that remains to be addressed concerns the microstructural properties that determine the individual expression of HFO and SICF in the sensorimotor cortices. One candidate is the degree of cortical myelination, because myelin determines 
crucial aspects of temporal processing in neuronal circuits, including conduction velocity, the synchronization of neuronal activity [17-19], and it is shaped by the firing rate of the action potentials [20,21]. Therefore, regional myelin content may sustain high-frequency phenomena and may set a limit on the maximal frequency that can be expressed in the cortical region.

In this study, we combined magnetic resonance imaging (MRI) of cortical myelination with recordings of HFO and SICF to test two hypotheses. We predicted that the individual expressions of HFO and SICF are tightly related in terms of periodicity and magnitude. We further hypothesized that the cortical myelin content in the sensorimotor cortex is related to the frequency or magnitude of HFO and SICF.

\section{Methods}

\subsection{Subjects}

Twenty-four subjects (14 female) aged 22-44 years (mean age \pm SEM: $33.13 \pm 2.64$ years) were enrolled in the study after giving their written informed consent. The experiments conformed to the Declaration of Helsinki and were approved by the Ethics Committee of the University of Naples Federico II, Italy (N. 100/17). None of the subjects had a history of neurological disease or received drugs interacting with the central nervous system at the time of the experiment. All the subjects were right-handed according to the Edinburgh Handedness Inventory $[22]$.

\subsection{Experimental procedures}

The experimental procedures are summarized in Fig.1. All participants underwent measurements of somatosensory HFO followed by paired-pulse TMS to obtain the individual SICF curve. Before starting with the TMS protocol, all subjects were screened for contraindications to TMS [23]. During the electrophysiology experiments, subjects were seated comfortably in a reclining chair with the right forearm placed in a prone position on the arm rest.

\subsection{Recordings of somatosensory cortical responses}


Peripheral electrical stimulation of the right median nerve was performed at the right wrist placing the anode on the crease and the cathode $2 \mathrm{~cm}$ proximal. Three thousand monophasic square-wave pulses of $200 \mu$ duration were delivered at a frequency of $5 \mathrm{~Hz}$ (Digitimer, Welwyn Garden City, UK). Stimulus intensity was adjusted to $120 \%$ of individual motor threshold, which is defined as the minimum stimulation intensity able to produce a small twitch of the abductor pollicis brevis muscle.

SEPs were recorded from scalp with $\mathrm{Ag}-\mathrm{AgCl}$ cup electrodes placed at $\mathrm{CP} 3$ (active channel) and at Fz (reference), according to the international 10-20 EEG montage system (Klem et al., 1999). We chose this montage because the bipolar parieto-frontal montage has been shown to be more sensitive to the BA3B component of HFO [24]. Cortical responses were recorded with a one-second-long trial at a sampling rate of 5 kHz using Signal software and CED 1401 hardware (Cambridge Electronic Design, Cambridge, UK). Preprocessing of the EEG data consisted in cubic-interpolation of the stimulus-related electric artefact from -0.2 to +6 ms [25] and exclusion of saturated trials. Subsequently, N20 peak latency and HFO were detected after bandpassed filtering, 3-2000 Hz and 400-800 Hz respectively (zero-phase second order Butterworth filter) and averaging the trials (Fig. 1A). We used the N20 latency to distinguish the two, subcortical and cortical, bursts present in HFO.

Further HFO analysis was based on findings from the studies from Baker [8] and Telenczuk [9], which had shown that the two central and highest peaks represent cortical population spiking of BA3B, in line with Shimazu's study [7]. We selected, for the subcortical and cortical sources, the two highest peaks before and after N20 respectively, which happened to be the closest peaks to N20 and to be always positive in our montage. Since the negative peaks, with respect to our montage, were probably ringing effects after filtering, we put them to zero. Our procedure yielded two HFO datasets that reflected the subcortical and cortical components and were comparable in shape to the SICF curve.

\subsection{Assessment of short interval intracortical facilitation (SICF)}

A biphasic TMS pulse was applied using a standard figure-of-eight coil (MC-B70 with outer diameter of each wing $97 \mathrm{~mm}$ ) connected to a high-power magnetic stimulator (MagPro X100, Medtronic, Denmark). The "hot spot” was defined as the scalp position over the left M1 where maximal motor evoked potentials (MEPs) was 
elicited in the contralateral first dorsal interosseous (FDI) muscle [26]. The signal was acquired via Ag-AgCl surface electrodes in a belly tendon montage, amplified, bandpass filtered (20 Hz-3 kHz) and digitized at a frequency of 5 kHz (Signal software and CED 1401 hardware, Cambridge Electronic Design, Cambridge, UK). We first determined the resting motor threshold (RMT), given in percentage of maximum stimulator output, as the minimum stimulus intensity that produced a MEP of $50 \mu \mathrm{V}$ in the relaxed right FDI muscle in at least 5 of 10 trials [27]. Finally, MEP1mV was determined as the stimulus intensity, which elicited in the resting FDI a MEP of $1 \mathrm{mV}$ on average in five consecutive trials.

The SICF curve was evaluated by applying paired-pulse TMS at fourteen inter-stimulus intervals (ISIs, Fig 1B), ranging from 1.0 to $3.6 \mathrm{~ms}$ and separated by steps of $0.2 \mathrm{~ms}$. The intensity of the first stimulus was set to MEP1mV and the second stimulus at 90\% RMT [11,28]. Fifteen stimuli for each ISI were tested in a randomized order. Mean MEP amplitude was calculated for each ISI and normalized to the MEP size elicited by the test stimulus alone. This yielded a SICF curve which covered the first and the second facilitatory peaks (Fig.1B).

\subsection{Structural MRI}

Participants underwent structural MRI of the brain immediately after the electrophysiological measurements had been completed. Three-dimensional MRI scans were acquired with a $3 \mathrm{~T}$ Trio Scanner equipped with an 8channel head coil (Siemens Healthineers, Erlangen, Germany). The acquisition protocol included a T1-weighted volume acquired using a 3D Magnetization Prepared RApid Acquisition Gradient Echo (MPRAGE) sequence consisting of 192 sagittal slices with $1 \mathrm{~mm}$ isotropic voxel resolution $(\mathrm{TR} / \mathrm{TE}=2300 / 2.96 \mathrm{~ms}$; TI = $1100 \mathrm{~ms}$; flip angle $=9^{\circ}$; matrix size $\left.=256 \times 240 \times 192\right)$ and a T2-weighted volume using a 3D turbo spin echo sequence with 176 sagittal slices and $1 \mathrm{~mm} 3$ voxel resolution $\left(\mathrm{TR} / \mathrm{TE}=3200 / 408 \mathrm{~ms}\right.$; flip angle $=120^{\circ}$; matrix size $=256 \times 258$ x176). MRI data of four subjects had to be excluded because of head movement related artefacts.

\subsection{Surface-based analysis of cortical morphology: thickness, curvature, and myelination}

Cortical reconstruction was performed with the FreeSurfer image analysis suite ver. 6.0.0 (http://surfer.nmr.mgh.harvard. edu/) (Fischl and Dale, 2000). The grey and white matter surfaces were defined by an automated brain segmentation process. If required, an experienced investigator (R.D.) manually corrected 
the automated segmentation, following the procedures outlined at https://surfer.nmr.mgh.harvard.edu/fswiki/Edits. The processes of surface extraction and inflation generated surface curvature, estimated from the mean curvature (or average of the principal curvatures) of the white matter surface [29] and cortical thickness, estimated at each point across the cortex by calculating the distance between the grey/white matter boundary and the cortical surface. Individual whole brain surface maps were smoothed with a $5 \mathrm{~mm}$ 2D Gaussian smoothing kernel [30] and the effect of surface curvature on cortical thickness was regressed out [31,32]. Individual curvature-corrected cortical thickness maps were registered to a common FreeSurfer template surface (fsaverage) for visualization and group analysis [33].

Procedures for mapping the myelin content of the cortical surface are described in detail by Glasser and Van Essen [34]. Briefly, a T1-weighted (T1w) and T2-weighted (T2w) image were registered to each other, and a ratio between the two images was calculated in each voxel of the brain, as a relative marker of myelin content (Fig. 1C). Specifically, the T1w image was brain-extracted and segmented into white matter and cerebro-spinal fluid using the FAST procedure [35] implemented in FSL [36]. The T2w image was brought into register with the T1w image using FSL flirt, employing a rigid registration with mutual information as cost function. At each voxel, the ratio between the normalized T1w image and the aligned normalized T2w image was calculated, which is believed to be contrast-sensitive to myelin [34]. To specifically examine only cortical voxels, the cortical ribbon was defined by voxels between white and pial surfaces, and the T1w/T2w ratios were sampled to each subject's individual ‘native’ central cortical surface by using cubic interpolation in MATLAB. We regressed out curvature since curvature-associated modulations can obscure or distort myelination changes due to other variations in cytoarchitectonic [37]. We finally smoothed the data applying a $5 \mathrm{~mm}$ 2D Gaussian smoothing kernel [30]. The individual maps were registered onto the FreeSurfer group template for visualization and group analysis (Fig 1D).

\section{Statistical analysis}

For both SICF and HFO, we extracted values of frequency and amplitude that were then used for statistical analysis. Centre of gravity $(\mathrm{CoG})$ of each peak was calculated for the peak latency and the distance of the two latencies was used as frequency measure. Regarding the amplitude measure, we computed the sum of the area 
under the curve (AUC) [38] obtained from the first and second peak (not normalized data). In the case of HFO, we considered the two highest peaks before and after N20 for early- and late-HFO respectively.

Before applying parametric statistical tests, the normal distribution of all variables was verified by means of a Kolmogorov and Smirnov test. Then, as a preliminary step, we evaluated the effect of ISI on SICF considering raw and not normalized MEPs values. In detail, one-way repeated measure analysis of variance (ANOVA-rm) was conducted with the within subject factor ISI (14 levels for SICF: 1.0 - $3.6 \mathrm{~ms}$ in $0.2 \mathrm{~ms}$ steps). Post hoc twotailed one-sample t-tests were conducted to identify ISI with significant MEP change with respect to Test stimulus. In addition, paired t-tests were used to compare AUC of the first (AUCpeak1) and the second SICF peak (AUCpeak2) and regarding HFO, to compare the frequency and AUC of the early vs late component. The second part of the analysis consisted of:

(i) Correlation of the temporal (i.e., frequency) and spatial (i.e., AUC) properties of the early and late components of HFO with frequency and area of SICF peaks. Specifically, for the frequency analysis, we compared the distance between the CoGs of the HFO peaks with that obtained from SICF peaks. For spatial analysis, we correlated the amplitudes as the sum of areas that subtended the peaks' curves for HFO and SICF.

(ii) Correlation of the temporal (frequency) and spatial (area) parameters of the early and late components of HFO, and of SICF with the individual estimates of cortical thickness (derived from T1-weighted MRI scans) and myelination (derived from T1w/T2w ratio mapping).

To visualize the spatial distribution of significant effects we computed surface-based analyses within the left sensory-motor cortex forming the hand knob. We defined our region-of-interest (RoI) as the cortical area covering the M1 (BA4), the adjacent dorsal Premotor cortex (dPM, BA6) till the bottom of the precentral sulcus (Dubbioso et al., 2021) and S1 (BA3 and part of BA1) till the top of the postcentral gyrus crown (Fig 1E). The multimodal parcellation atlas derived from the Human Connectome Project [31] was used to visualize the border between premotor, motor, and somatosensory areas.

To visualize the spatial distribution of significant effects we computed surface-based analyses within the RoI on the FsAverage template by using Freesurfer software [33]. These analyses were performed vertex-wise, followed by cluster-wise corrections for multiple comparisons based on permutation method [39](cluster-determining 
threshold: $\mathrm{p}<0.01$, cluster-wise $\mathrm{p}<0.05$ ) [33]. Age at the time of MRI and sex were included in the model as nuisance variables.

The alpha inflation due to multiple comparisons was faced according to the Bonferroni procedure.

Descriptive statistics are reported as mean \pm standard error of the mean (SEM). All statistical analyses used IBM SPSS Statistics software (Version 22 for Windows, New York City, USA).

\section{Results}

\subsection{Short-latency intracortical facilitation (SICF)}

The paired-pulse measurements showed consistent SICF in all individuals. SICF measurements yielded a main effect of ISI on mean MEP amplitude $\left(\mathrm{F}_{(4.419,101.644)}=8.393, \mathrm{p}<0.001\right)$. Mean MEP amplitude elicited by pairedpulse stimulation were larger at ISIs of 1.2-1.8 (peak1) and 2.2-3.0 ms (peak2) relative to single-pulse TMS (all paired t-tests: $\mathrm{p}<0.033$ ). These peaks were separated by a trough at ISI of $2 \mathrm{~ms}$, where paired stimulation had no significant effect on MEP size (paired t-test: $\mathrm{p}=0.724$ ). The magnitude of these two peaks was expressed as AUC, the AUC of the first peak was significantly higher with respect to the second one (AUCpeak1= $2.14 \pm$ 0.15 vs AUCpeak2= $1.88 \pm 0.14, \mathrm{p}=0.011)$.

\subsection{High-frequency cortical oscillations (HFO)}

Subcortical and cortical HFO did not differ regarding peak-to-peak distance $(p=0.873): 1.54 \pm 0.02$ ms (earlyHFO) vs $1.55 \pm 0.06 \mathrm{~ms}$ (late-HFO), nor regarding the amplitude ( $\mathrm{p}=0.511): 0.35 \pm 0.04 \mu \mathrm{V}^{2} \times 10^{-3}$ (early-HFO) vs $0.33 \pm 0.04 \mu \mathrm{V}^{2} \times 10^{-3}$ (late-HFO).

\subsection{Relationship between SICF and HFO}

The individual expression of late-HFO and SICF were tightly correlated (Fig. 2). The individual frequencies of HFO and SICF, i.e., the interval between the first and second peak of cortical late-HFO and SICF, showed a positive linear relationship ( $\mathrm{r}=0.703, \mathrm{p}<0.001$, Fig. 2A). Conversely, the response amplitudes of cortical lateHFOs and SICF, indexed by the area under the curve, showed a negative linear relation $(r=-0.613, p=0.001$, Fig. 2B). No significant correlation was observed between the frequencies $(r=0.016, p=0.941)$ and amplitudes ( $r=$ - 
0.394, $\mathrm{p}=0.057$ ) of sub-cortical early-HFOs and SICF. Significance level was set at $\mathrm{p}<0.006$ after correction for multiple comparisons.

\subsection{Relationship between cortical myelination and cortical high-frequency responses patterns}

The individual peak-to-peak intervals of both SICF and cortical late-HFOs showed voxel-based correlation with the myelin content in the S1 and with the crown of precentral gyrus (Fig. 3A and 3C). After cluster-based multiple comparison correction, surface-based correlation analyses revealed that the T1w/T2w ratio correlated with the individual frequency of cortical HFO in a cluster located posterior to the central sulcus, in the S1 (Fig. 3A; peak correlation at $\mathrm{x}, \mathrm{y}, \mathrm{z}=-35.3,-30,52.8)$. For SICF, the correlation between the peak-to-peak interval of SICF and cortical myelin content was significant in a cluster covering mainly S1 along the whole hand knob (Fig. 3C; peak correlation at $\mathrm{x}, \mathrm{y}, \mathrm{z}=-50.3,-17.8,51.2)$. Correlation analysis did not disclose any significant cluster in S1 or M1 that displayed a relation between the degree of cortical myelination and the amplitude of late-HFO or SICF. As for other cases, the subcortical early-HFO did not show significant correlation with myelin content. We also found no significant cluster when testing for a linear relationship between cortical thickness of the left S1 or M1 and the temporal and spatial properties of early-HFO, late-HFO and SICF.

\section{Discussion}

We found that the individual frequencies and magnitudes of late-HFO and SICF were tightly correlated. The intervals between the first and second peak of cortical HFO and SICF showed a positive linear relationship, while their magnitudes had a negative relation. The higher the cortical myelin content, the shorter were the interpeak intervals of late-HFO and SICF, but there was no relation between amplitudes and myelin. Early-HFO, that are of subcortical origin and used in our study as a control condition, were not correlated either with SICF or with myelin content in the cortex.

\subsection{High-frequency responses in the S1 and M1 are mutually related}

The high-frequency response in the S1 evoked by peripheral electrical stimulation (i.e., late-HFO) was positively related to the rhythmicity of SICF evoked in M1 with paired-pulse TMS (Fig 2A). The generators of late-HFO 
activity are bursting pyramidal neurons in BA3B [8,9], most likely the thick tufted pyramidal neurons from layer 5b that are known to be activated by passive touch [40,41]. In fact, not only they are intrinsic bursting neurons that exhibit a bursting frequency compatible with HFO, but they also have adequate structure and geometry to be detected by EEG: they are well aligned in a parallel fashion with dendrites spreading from the lower part of layer 5 up to layer 1 on the cortical surface [42-44]. Thick tufted neurons are pyramidal-tract (PT) neurons which have cortico-subcortical, mainly cortico-spinal axonal projections [45,46]. Interestingly, PT neurons display high-frequency descending axonal activity in response to cortical stimulation, defined as indirect waves (iwaves), as observed after local cortical stimulation of the sensory and the motor primary areas [12]. And SICF is thought to provide a non-invasive assessment of i-waves in human M1 [10,12].

\subsection{Inverse relation of the magnitude of sensory and motor high-frequency responses}

The individual response magnitudes of late-HFO and SICF showed an inverse relationship of areas underlying the peaks of the responses (Fig 2B). Pharmacological and TMS studies showed that both protocols are dependent on GABA-ergic inhibitory circuits. Specifically, it has been suggested that late-HFO reflect the activity of inhibitory interneurons that produce feedforward inhibition of pyramidal neurons [4], and analogously the high frequency repetitive discharge of the corticospinal axons in SICF is the result of recruitment of GABA(A)-ergic interneurons together with highly synchronized excitatory neurons [16]. Pharmacological studies showed increased SICF when a higher presynaptic release of glutamate was induced [47] and the facilitation was inhibited by positive allosteric modulators of GABA(A) receptor [48,49], but not GABA(B) agonist [48]. Importantly, these modulations are related only to the later I-wave peaks. Similarly, GABA(A)-receptor antagonist, bicuculline methiodide, increased the number of peaks of late-HFO [50]. This fits again with the characteristics of intrinsic bursting thick tufted PT neurons. In fact, GABA(A) inhibitory interneurons [51] can promote pyramidal neurons from single spike to bursting neurons [52,53], keeping the first peak stable and influencing only later components of the burst.

Our study was not designed to clarify the mechanism behind the inverse relationship between the late-HFO response generated in S1 and SICF amplitude in M1. Our findings might be explained by the application of two 
different types of stimulation that could have opposite effects on the GABA-ergic circuits, or by unexplored inhibition/excitation balances between S1 and M1. In both cases, this finding warrants further study.

\subsection{The frequency of high-frequency cortical responses is related to cortical myelin content}

MRI-based cortical myelin mapping identified cortical clusters in S1 where regional myelin content correlated positively with the individual frequency of late-HFO and SICF peaks (Fig 3). The higher the frequencies, the stronger was regional axonal myelination. This finding is compatible with the notion that the ability to generate faster rhythmic activity requires a higher myelin content [19]. The finding is also in good agreement with previous work showing axonal myelination is dynamically shaped by the amount of its electrical activity [20,21]. We also expected to find clusters in M1, since SICF is generated in the motor cortex [12], but significant clusters were confined to the sensory cortex in the postcentral gyrus. The voxel-based correlation map (Fig. 3) showed also spots on the crown of the precentral gyrus, where TMS produces the strongest electrical field [32,54], but the correlation didn't survive after cluster-based multiple comparisons. This might be due to the structural inhomogeneity of the motor cortex, where, contrary to other brain regions, layer 5b also contains large Betz cells [55], which likely introduce inter-individual variability in the estimation of regional myelin related to high frequency activity. In fact, Betz cells display a different shape, dendrites, and size compared to the surrounding pyramidal neurons [56]. In addition, they are not distributed homogenously in the precentral gyrus, as they show a mediolateral gradient with a cluster in the precentral hand knob, the region of our interest [57]. Therefore, a larger sample size may be needed to reveal a significant correlation between regional myelin content in precentral M1 and the individual frequency of late-HFO and SICF peaks. This issue remains to be addressed in more detail in future studies.

\section{Conclusions}

Our study sheds new light on high-frequency responses in the primary sensory and motor cortices in humans. We showed a strong link between late-HFO and SICF. Both cortical phenomena may reflect the activity of intrinsic bursts of thick tufted PT neurons that are generated in strong functional interaction with GABA-ergic inhibitory interneurons. Our results also suggest a strong link between the rhythmicity of the cortical high-frequency 
responses and regional myelin content, underscoring the importance of regional myelination for the precise timing of neuronal activity in the human cortex. The results of this study also have important implications for the clinical neurophysiological assessment of cortical dysfunction. For instance, multiple sclerosis is characterized by a reduction of cortical myelin content [58,59] and slower high-frequency bursts [60], emphasizing the dependency of high-frequency activity on intact cortical myelination.

\section{References}

[1] Cruccu G, Aminoff MJ, Curio G, Guerit JM, Kakigi R, Mauguiere F, et al. Recommendations for the clinical use of somatosensory-evoked potentials. Clin Neurophysiol 2008;119:1705-19. doi:10.1016/j.clinph.2008.03.016.

[2] Ozaki I, Suzuki C, Yaegashi Y, Baba M, Muneo Matsunaga, Hashimoto I. High frequency oscillations in early cortical somatosensory evoked potentials. Electroencephalogr Clin Neurophysiol - Evoked Potentials 1998;108:536-42. doi:10.1016/S0168-5597(98)00032-X.

[3] Buzsáki G, Draguhn A. Neuronal oscillations in cortical networks. Science 2004;304:1926-9. doi:10.1126/science.1099745.

[4] Ozaki I, Hashimoto I. Exploring the physiology and function of high-frequency oscillations (HFOs) from the somatosensory cortex. Clin Neurophysiol 2011;122:1908-23. doi:10.1016/j.clinph.2011.05.023.

[5] Buzsáki G, Silva FL da. High frequency oscillations in the intact brain. Prog Neurobiol 2012;98:241-9. doi:10.1016/j.pneurobio.2012.02.004.

[6] Ritter P, Freyer F, Curio G, Villringer A. High-frequency $(600 \mathrm{~Hz})$ population spikes in human EEG delineate thalamic and cortical fMRI activation sites. Neuroimage 2008;42:483-90. doi:10.1016/j.neuroimage.2008.05.026.

[7] Shimazu H, Kaji R, Tsujimoto T, Kohara N, Ikeda A, Kimura J, et al. High-frequency SEP components generated in the somatosensory cortex of the monkey. Neuroreport 2000;11:2821-6. doi:10.1097/00001756-200008210-00042. 
[8] Baker SN, Curio G, Lemon RN. EEG oscillations at $600 \mathrm{~Hz}$ are macroscopic markers for cortical spike bursts. J Physiol 2003;550:529-34. doi:10.1113/jphysiol.2003.045674.

[9] Telenczuk B, Baker SN, Herz AVM, Curio G. High-frequency EEG covaries with spike burst patterns detected in cortical neurons. J Neurophysiol 2011;105:2951-9. doi:10.1152/jn.00327.2010.

[10] Tokimura H, Ridding MC, Tokimura Y, Amassian VE, Rothwell JC. Short latency facilitation between pairs of threshold magnetic stimuli applied to human motor cortex. Electroencephalogr Clin Neurophysiol - Electromyogr Mot Control 1996;101:263-72. doi:10.1016/0924-980X(96)95664-7.

[11] Ziemann U, Tergau F, Wassermann EM, Wischer S, Hildebrandt J, Paulus W. Demonstration of facilitatory I wave interaction in the human motor cortex by paired transcranial magnetic stimulation. $\mathrm{J}$ Physiol 1998;511:181-90. doi:10.1111/j.1469-7793.1998.181bi.x.

[12] Patton HD, Amassian VE. Single and multiple-unit analysis of cortical stage of pyramidal tract activation. J Neurophysiol 1954;17:345-63. doi:10.1152/jn.1954.17.4.345.

[13] Di Lazzaro V, Oliviero A, Profice P, Saturno E, Pilato F, Insola A, et al. Comparison of descending volleys evoked by transcranial magnetic and electric stimulation in conscious humans.

Electroencephalogr Clin Neurophysiol - Electromyogr Mot Control 1998;109:397-401. doi:10.1016/S0924-980X(98)00038-1.

[14] Avanzino L, Teo JTH, Rothwell JC. Intracortical circuits modulate transcallosal inhibition in humans. J Physiol 2007;583:99-114. doi:10.1113/jphysiol.2007.134510.

[15] Ziemann U. I-waves in motor cortex revisited. Exp Brain Res 2020;238:1601-10. doi:10.1007/s00221020-05764-4.

[16] Di Lazzaro V, Ziemann U. The contribution of transcranial magnetic stimulation in the functional evaluation of microcircuits in human motor cortex. Front Neural Circuits 2013;7:1-9. doi:10.3389/fncir.2013.00018.

[17] Seidl AH, Rubel EW, Harris DM. Mechanisms for adjusting interaural time differences to achieve binaural coincidence detection. J Neurosci 2010;30:70-80. doi:10.1523/JNEUROSCI.3464-09.2010.

[18] Ford MC, Alexandrova O, Cossell L, Stange-Marten A, Sinclair J, Kopp-Scheinpflug C, et al. Tuning of Ranvier node and internode properties in myelinated axons to adjust action potential timing. Nat 
Commun 2015;6:8073. doi:10.1038/ncomms9073.

[19] Pajevic S, Basser PJ, Fields RD. Role of myelin plasticity in oscillations and synchrony of neuronal activity. Neuroscience 2014;276:135-47. doi:10.1016/j.neuroscience.2013.11.007.

[20] Demerens C, Stankoff B, Logak M, Anglade P, Allinquant B, Couraud F, et al. Induction of myelination in the central nervous system by electrical activity. Proc Natl Acad Sci U S A 1996;93:9887-92. doi:10.1073/pnas.93.18.9887.

[21] Stevens B, Porta S, Haak LL, Gallo V, Fields RD. Adenosine: A neuron-glial transmitter promoting myelination in the CNS in response to action potentials. Neuron 2002;36:855-68. doi:10.1016/S08966273(02)01067-X.

[22] Oldfield RC. The assessment and analysis of handedness: The Edinburgh inventory. Neuropsychologia 1971;9:97-113. doi:10.1016/0028-3932(71)90067-4.

[23] Rossi S, Antal A, Bestmann S, Bikson M, Brewer C, Brockmöller J, et al. Safety and recommendations for TMS use in healthy subjects and patient populations, with updates on training, ethical and regulatory issues: Expert Guidelines. Clin Neurophysiol 2021;132:269-306. doi:10.1016/j.clinph.2020.10.003.

[24] Klostermann F, Gobbele R, Buchner H, Siedenberg R, Curio G. Differential gating of slow postsynaptic and high-frequency spike-like components in human somatosensory evoked potentials under isometric motor interference. Brain Res 2001;922:95-103. doi:10.1016/S0006-8993(01)03157-2.

[25] Katayama T, Suppa A, Rothwell JC. Somatosensory evoked potentials and high frequency oscillations are differently modulated by theta burst stimulation over primary somatosensory cortex in humans. Clin Neurophysiol 2010;121:2097-103. doi:10.1016/j.clinph.2010.05.014.

[26] Groppa S, Oliviero A, Eisen A, Quartarone A, Cohen LG, Mall V, et al. A practical guide to diagnostic transcranial magnetic stimulation: Report of an IFCN committee. Clin Neurophysiol 2012;123:858-82. doi:10.1016/j.clinph.2012.01.010.

[27] Rossini PM, Burke D, Chen R, Cohen LG, Daskalakis Z, Di Iorio R, et al. Non-invasive electrical and magnetic stimulation of the brain, spinal cord, roots and peripheral nerves: Basic principles and procedures for routine clinical and research application: An updated report from an I.F.C.N. Committee. Clin Neurophysiol 2015;126:1071-107. 
[28] Hanajima R, Ugawa Y, Terao Y, Enomoto H, Shiio Y, Mochizuki H, et al. Mechanisms of intracortical Iwave facilitation elicited with paired-pulse magnetic stimulation in humans. J Physiol 2002;538:253-61. doi:10.1113/jphysiol.2001.013094.

[29] Pienaar R, Fischl B, Caviness V, Makris N, Grant PE. A methodology for analyzing curvature in the developing brain from preterm to adult. Int J Imaging Syst Technol 2008;18:42-68. doi:10.1002/ima.20138.

[30] Fischl B, Dale AM. Measuring the thickness of the human cerebral cortex from magnetic resonance images. Proc Natl Acad Sci 2000;97:11050-5. doi:10.1073/pnas.200033797.

[31] Glasser MF, Coalson TS, Robinson EC, Hacker CD, Harwell J, Yacoub E, et al. A multi-modal parcellation of human cerebral cortex. Nature 2016;536:171-8. doi:10.1038/nature18933.

[32] Dubbioso R, Madsen KH, Thielscher A, Siebner HR. The myelin content of the human precentral hand knob reflects interindividual differences in manual motor control at the physiological and behavioral level. J Neurosci 2021;41:3163-79. doi:10.1523/JNEUROSCI.0390-20.2021.

[33] Fischl B. FreeSurfer. Neuroimage 2012;62:774-81. doi:10.1016/j.neuroimage.2012.01.021.

[34] Glasser MF, van Essen DC. Mapping human cortical areas in vivo based on myelin content as revealed by T1- and T2-weighted MRI. J Neurosci 2011;31:11597-616. doi:10.1523/JNEUROSCI.2180-11.2011.

[35] Zhang Y, Brady M, Smith S. Segmentation of brain MR images through a hidden Markov random field model and the expectation-maximization algorithm. IEEE Trans Med Imaging 2001;20:45-57. doi:10.1109/42.906424.

[36] Jenkinson M, Beckmann CF, Behrens TEJ, Woolrich MW, Smith SM. FSL. Neuroimage 2012;62:782-90.

[37] Annese J, Pitiot A, Dinov ID, Toga AW. A myelo-architectonic method for the structural classification of cortical areas. Neuroimage 2004;21:15-26. doi:10.1016/j.neuroimage.2003.08.024.

[38] Dubbioso R, Raffin E, Karabanov A, Thielscher A, Siebner HR. Centre-surround organization of fast sensorimotor integration in human motor hand area. Neuroimage 2017;158:37-47. doi:10.1016/j.neuroimage.2017.06.063.

[39] Hayasaka S, Phan KL, Liberzon I, Worsley KJ, Nichols TE. Nonstationary cluster-size inference with random field and permutation methods. Neuroimage 2004;22:676-187. 
doi:10.1016/j.neuroimage.2004.01.041.

[40] Oberlaender M, Boudewijns ZSRM, Kleele T, Mansvelder HD, Sakmann B, De Kock CPJ. Threedimensional axon morphologies of individual layer 5 neurons indicate cell type-specific intracortical pathways for whisker motion and touch. Proc Natl Acad Sci U S A 2011;108:4188-93. doi:10.1073/pnas.1100647108.

[41] de Kock CPJ, Pie J, Pieneman AW, Mease RA, Bast A, Guest JM, et al. High-frequency burst spiking in layer 5 thick-tufted pyramids of rat primary somatosensory cortex encodes exploratory touch. Commun Biol 2021;4:1-14. doi:10.1038/s42003-021-02241-8.

[42] Chagnac-Amitai Y, Connors BW. Synchronized excitation and inhibition driven by intrinsically bursting neurons in neocortex. J Neurophysiol 1989;62:1149-62. doi:10.1152/jn.1989.62.5.1149.

[43] Chagnac-Amitai Y, Luhmann HJ, Prince DA. Burst generating and regular spiking layer 5 pyramidal neurons of rat neocortex have different morphological features. J Comp Neurol 1990;296:598-613. doi:10.1002/cne.902960407.

[44] Connors BW, Gutnick MJ. Intrinsic firing patterns of diverse neocortical neurons. Trends Neurosci 1990;13:99-104.

[45] Gao WJ, Zheng ZH. Target-specific differences in somatodendritic morphology of layer V pyramidal neurons in rat motor cortex. J Comp Neurol 2004;476:174-85. doi:10.1002/cne.20224.

[46] Anderson CT, Sheets PL, Kiritani T, Shepherd GMG. Sublayer-specific microcircuits of corticospinal and corticostriatal neurons in motor cortex. Nat Neurosci 2010;13:739-44. doi:10.1038/nn.2538.

[47] Mori F, Ribolsi M, Kusayanagi H, Monteleone F, Mantovani V, Buttari F, et al. TRPV1 channels regulate cortical excitability in humans. J Neurosci 2012;32:873-9. doi:10.1523/JNEUROSCI.2531-11.2012.

[48] Ziemann U, Tergau F, Wischer S, Hildebrandt J, Paulus W. Pharmacological control of facilitatory I-wave interaction in the human motor cortex. A paired transcranial magnetic stimulation study. Electroencephalogr Clin Neurophysiol - Electromyogr Mot Control 1998;109:321-30. doi:10.1016/S0924-980X(98)00023-X.

[49] Ilić T V., Meintzschel F, Cleff U, Ruge D, Kessler KR, Ziemann U. Short-interval paired-pulse inhibition and facilitation of human motor cortex: the dimension of stimulus intensity. J Physiol 2002;545:153-67. 
[50] Jones MS, Barth DS. Effects of Bicuculline Methiodide on Fast (\&gt;200 Hz) Electrical Oscillations in Rat Somatosensory Cortex. J Neurophysiol 2002;88:1016-25. doi:10.1152/jn.2002.88.2.1016.

[51] Murayama M, Pérez-Garci E, Nevian T, Bock T, Senn W, Larkum ME. Dendritic encoding of sensory stimuli controlled by deep cortical interneurons. Nature 2009;457:1137-41. doi:10.1038/nature07663.

[52] Williams SR, Stuart GJ. Mechanisms and consequences of action potential burst firing in rat neocortical pyramidal neurons. J Physiol 1999;521:467-82. doi:10.1111/j.1469-7793.1999.00467.x.

[53] Silberberg G, Markram H. Disynaptic Inhibition between Neocortical Pyramidal Cells Mediated by Martinotti Cells. Neuron 2007;53:735-46. doi:10.1016/j.neuron.2007.02.012.

[54] Bungert A, Antunes A, Espenhahn S, Thielscher A. Where does TMS Stimulate the Motor Cortex? Combining Electrophysiological Measurements and Realistic Field Estimates to Reveal the Affected Cortex Position. Cereb Cortex 2017;27. doi:10.1093/cercor/bhw292.

[55] Betz K. Anatomischer Nachweis zweier Gehirnzentra. Zentralbl Med Wiss 1874;12:595-9.

[56] Meyer G. Forms and spatial arrangement of neurons in the primary motor cortex of man. J Comp Neurol 1987;262:402-28. doi:10.1002/cne.902620306.

[57] Rivara CB, Sherwood CC, Bouras C, Hof PR. Stereologic characterization and spatial distribution patterns of Betz cells in the human primary motor cortex. Anat Rec - Part A Discov Mol Cell Evol Biol 2003;270:137-51. doi:10.1002/ar.a.10015.

[58] Lucchinetti CF, Popescu BFG, Bunyan RF, Moll NM, Roemer SF, Lassmann H, et al. Inflammatory cortical demyelination in early multiple sclerosis. N Engl J Med 2011;365:2188-97. doi:10.1056/NEJMoa1100648.

[59] Righart R, Biberacher V, Jonkman LE, Klaver R, Schmidt P, Buck D, et al. Cortical pathology in multiple sclerosis detected by the T1/T2-weighted ratio from routine magnetic resonance imaging. Ann Neurol 2017;82:519-29. doi:10.1002/ana.25020.

[60] Rossini PM, Basciani M, Di Stefano E, Febbo A, Mercuri N. Short-latency scalp somatosensory evoked potentials and central spine to scalp propagation characteristics during peroneal and median nerve stimulation in multiple sclerosis. Electroencephalogr Clin Neurophysiol 1985;60:197-206. doi:10.1016/0013-4694(85)90031-8. 


\section{Figure 1}

Experimental set-up. (A) Somatosensory evoked potential, SEP (dotted line) and high frequency oscillations, HFO (continuous line) after filtering and averaging EEG signal from CP3 active and Fz reference electrodes (light blue circles). HFO are divided in the early and the late components occurring before and after N20 peak respectively. (B) SICF curve over M1 at interstimulus intervals between 1.0 - $3.6 \mathrm{~ms}$, covering the early facilitatory peaks (Peak 1 and Peak 2). (C) From left to right: T1w and T2w MRI scans with the white matter (green line) and cortical pial surfaces (yellow line); T1w/T2w ratios are estimated by dividing the T1w by the T2w image and the values are sampled on the mid-thickness surface, which is positioned halfway in-between the white matter surface and cortical pial surface. (D) Cortical myelination amount across different cortical regions. (E) Region of Interest used for surface analysis. M1 = primary motor cortex; dPM = dorsal Premotor cortex; S1 $=$ primary somatosensory cortex.

\section{Figure 2}

\section{Relationship between somatosensory cortical high frequency oscillation (late HFO) and SICF}

(A) Significant positive correlation between the interpeak interval of late-HFO and that elicited by SICF. (B) Significant negative correlation between the Area under the curve (AUC) of the first and second SICF peak and the AUC of the late component of HFO.

Significant correlations are indicated in bold and continuous lines. Significant p value $<0.006$.

\section{Figure 3}

\section{Relationship between cortical myelination and somatosensory high frequency oscillation (late-HFO) and SICF}

(A) Surface-rendered statistical parametric map shows voxels with a negative linear relationship between cortical myelination and the inter-peak interval of late-HFO ( $\mathrm{p}$ uncorrected $<0.05$, left panel). The significant cluster is located in the primary somatosensory cortex (BA3) of the hand knob region peaking at $\mathrm{x}-, \mathrm{y}-, \mathrm{z}-$ coordinates $-35,-30,53$ (cluster-wise corrected, $p=0.039$, right panel). (B) Linear correlation between the T1w/T2w estimated myelination in the significant cluster and the interpeak interval of the late-HFO. (C) 
Surface-rendered statistical parametric maps show voxels with a negative relationship between cortical myelination and the inter-peak interval of SICF mainly in the primary somatosensory cortex and the crown of precentral gyrus (p uncorrected $<0.05$, left panel). The significant cluster is located at the hand knob region in the primary somatosensory cortex region peaking at x-,y-,z-coordinates -50, -18, 51 (cluster-wise corrected, $\mathrm{p}=$ 0.006). (D) Linear correlation between the $\mathrm{T} 1 \mathrm{w} / \mathrm{T} 2 \mathrm{w}$ estimated myelination in the significant cluster and the interpeak interval of the SICF. The region of interest (ROI) is highlighted in all brain maps. 
A

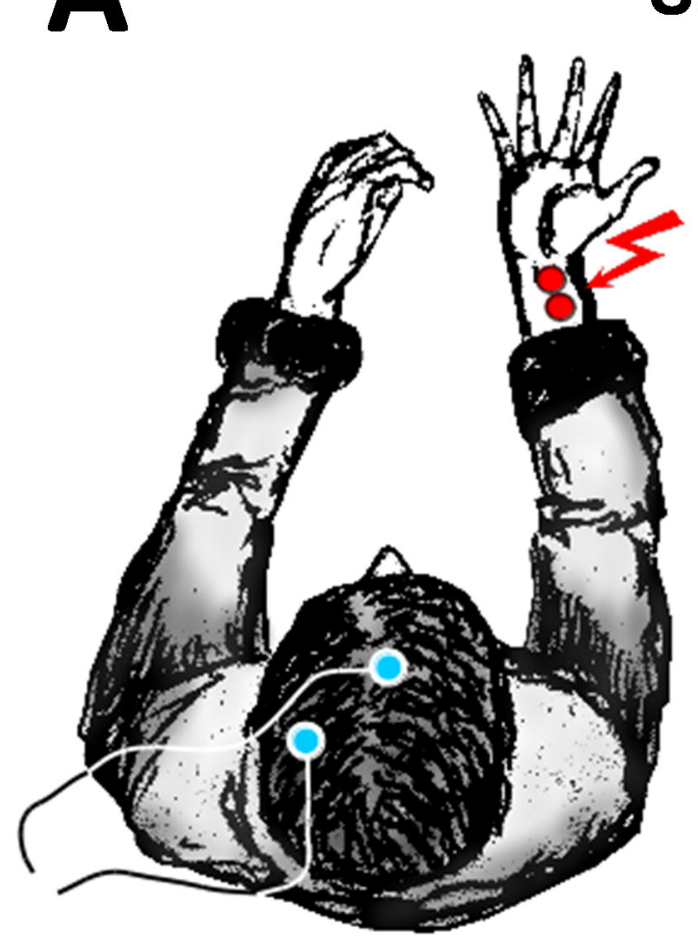

SOMATOSENSORY HIGH FREQUENCY

OSCILLATIONS

( $\mathrm{H}_{\mathrm{N}} \mathrm{O}$ )

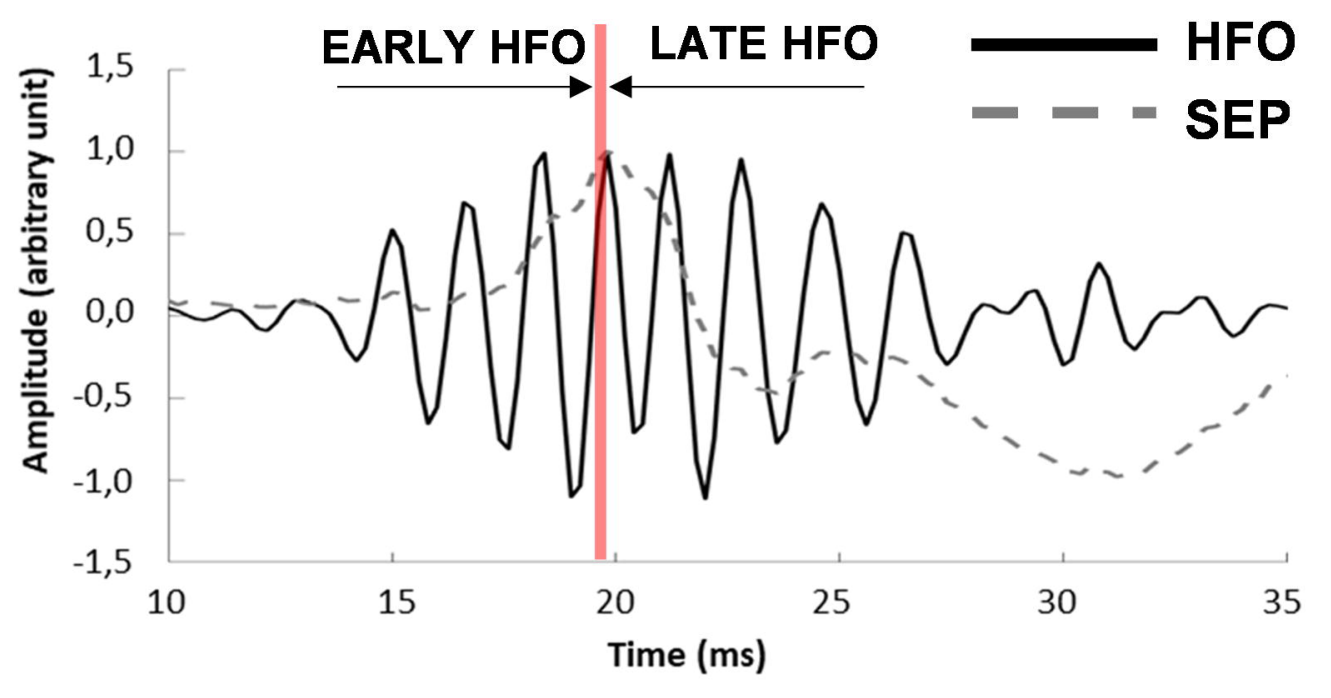

Recording Stimulating electrodes electrodes

C T1w brain scan

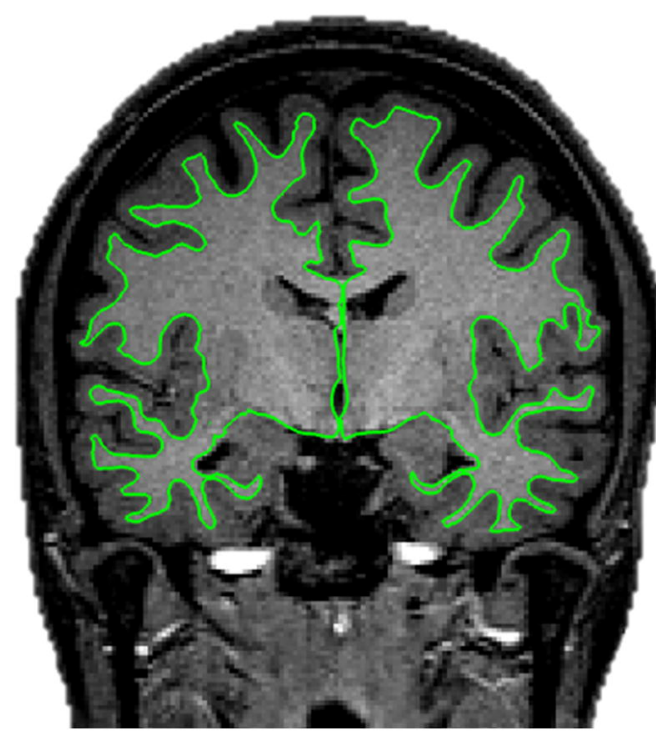

T2w brain scan

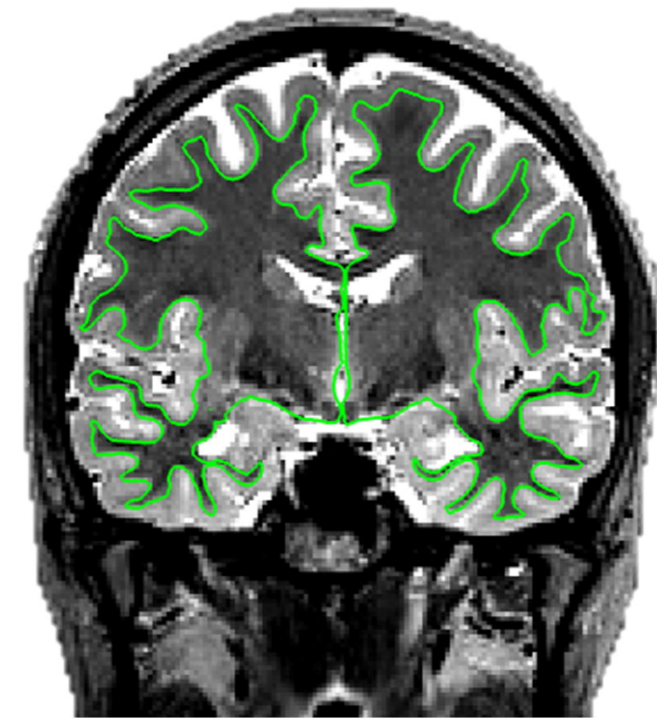

$\mathrm{T} 1 \mathrm{w} / \mathrm{T} 2 \mathrm{w}$ ratio

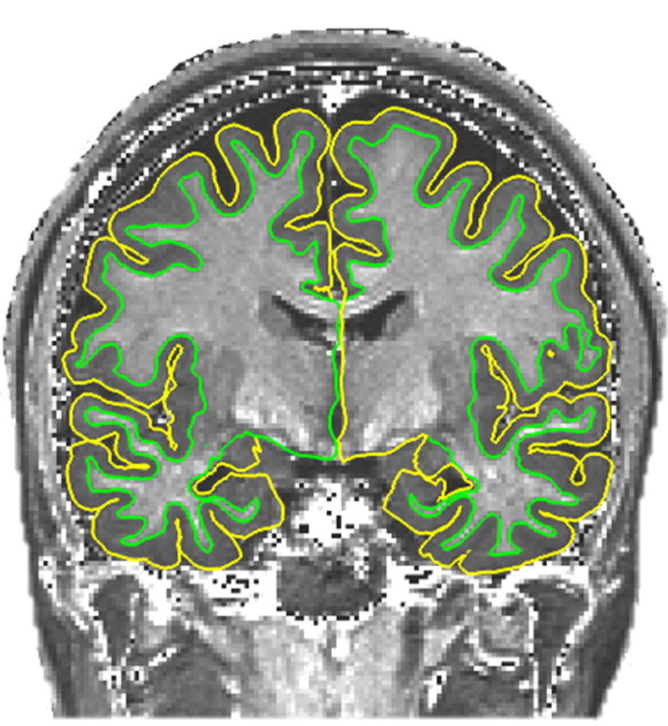

B

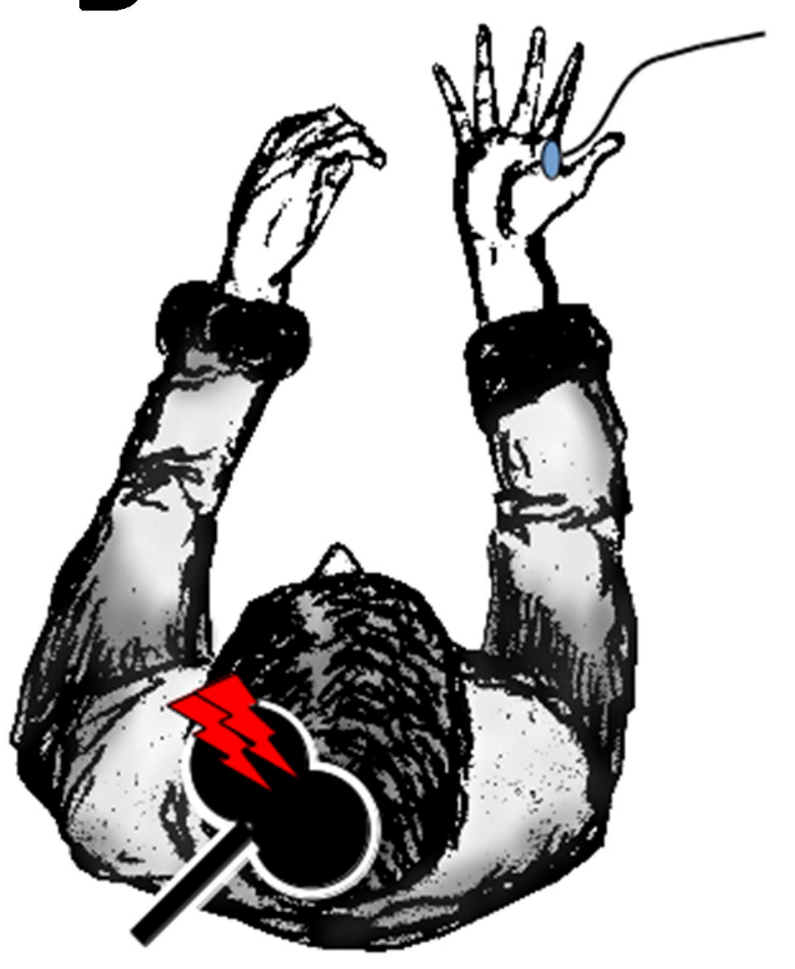

SHORT INTERVAL INTRACORTICAL FACILITATION (SICF)

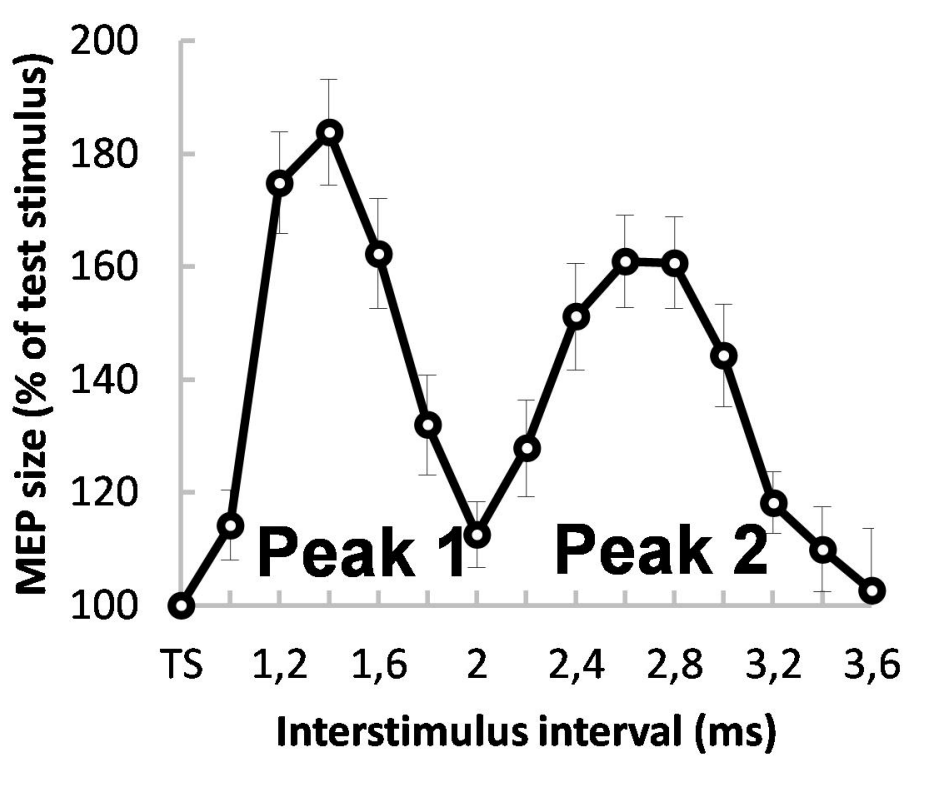

DURFACE MYELINATION MAPE
(T1w/T2w ratio)

Primary motor cortex (M1) Primary somatosensory cortex (S1)

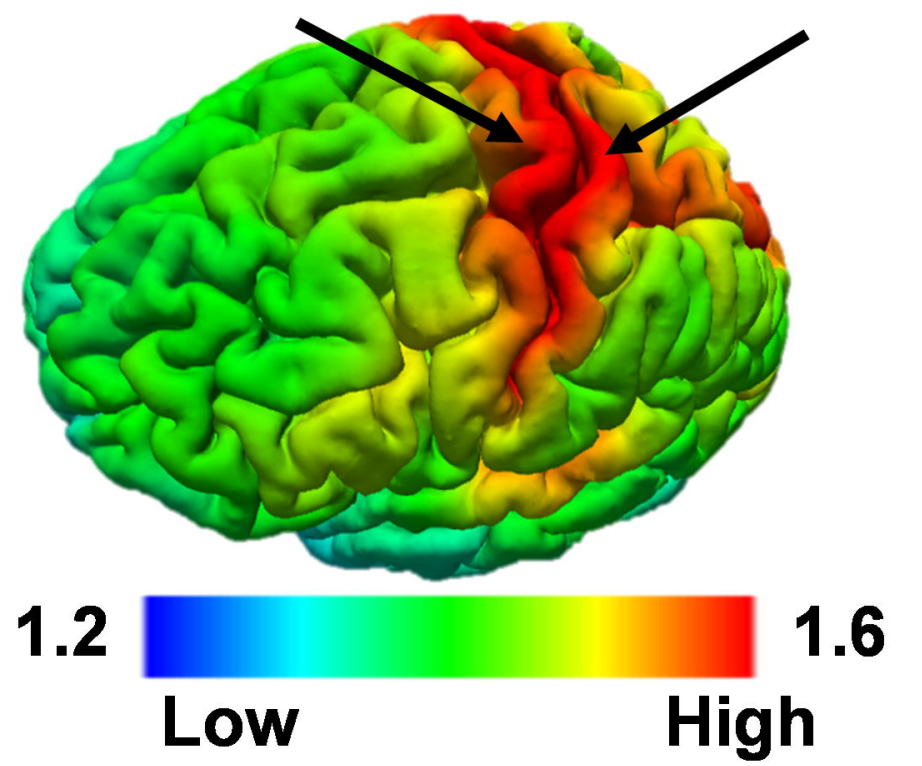

REGION OF INTEREST

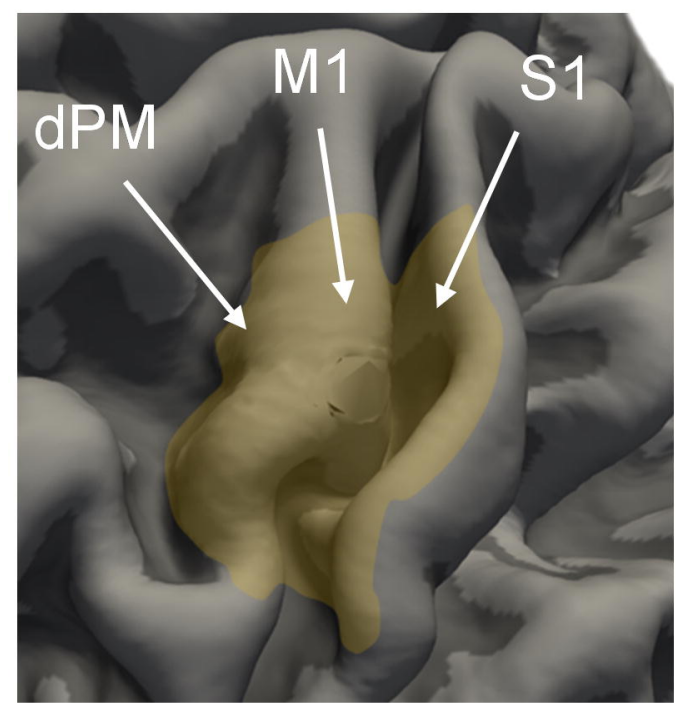


A

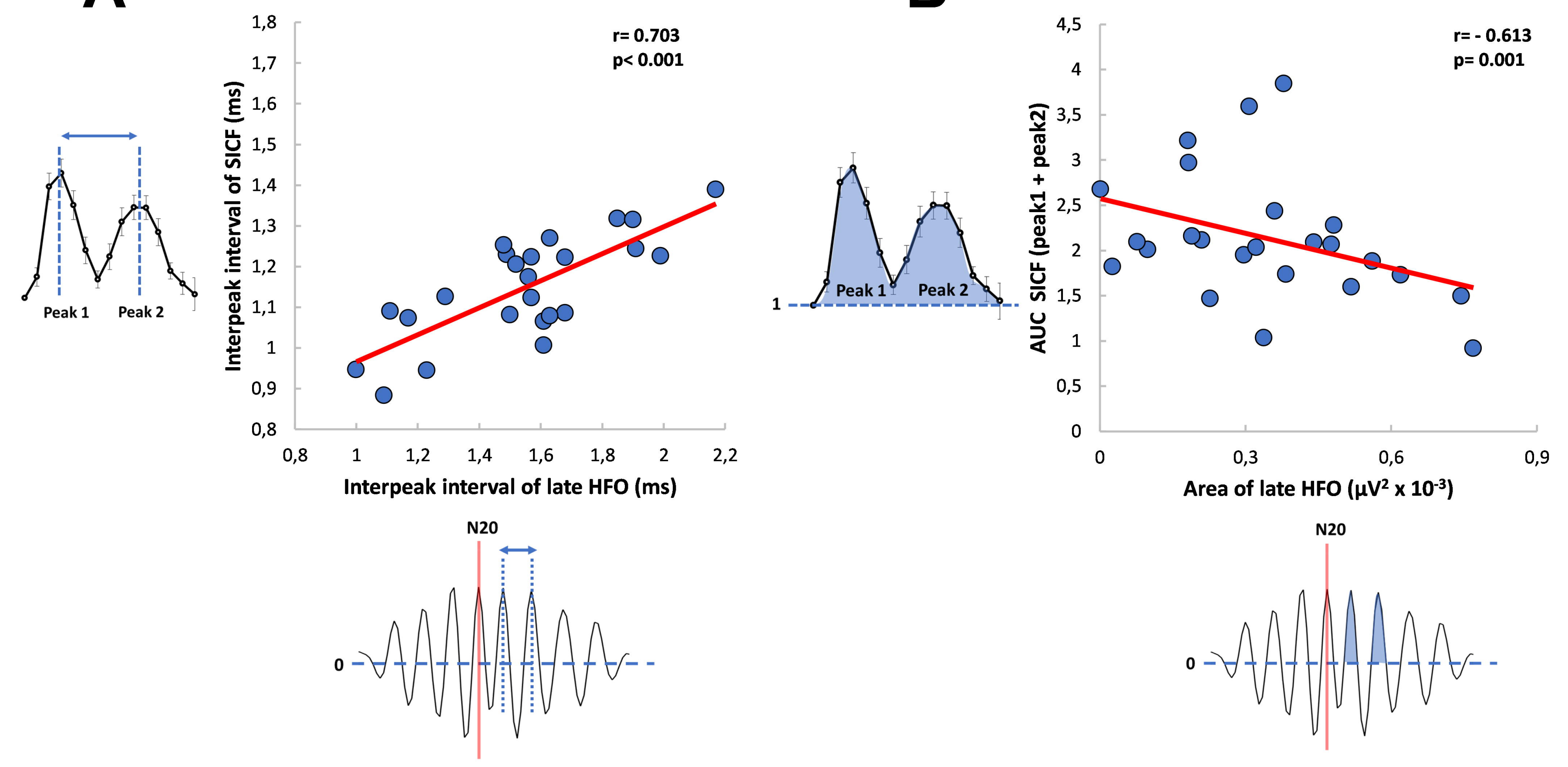


A

Uncorrected Surface correlation analysis

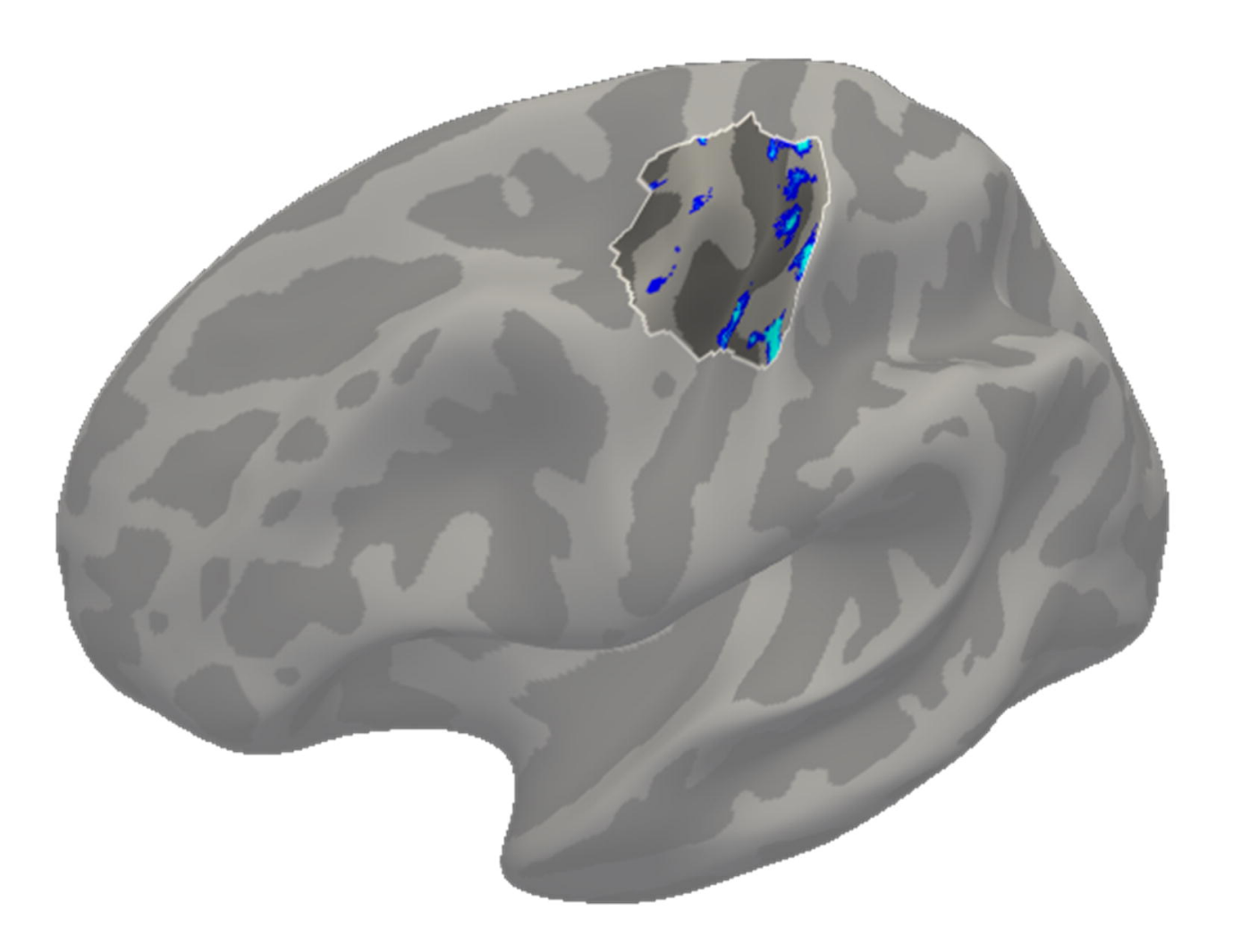

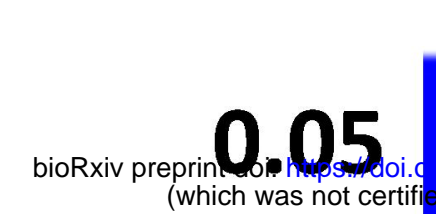

C

\section{Uncorrected Surface} correlation analysis

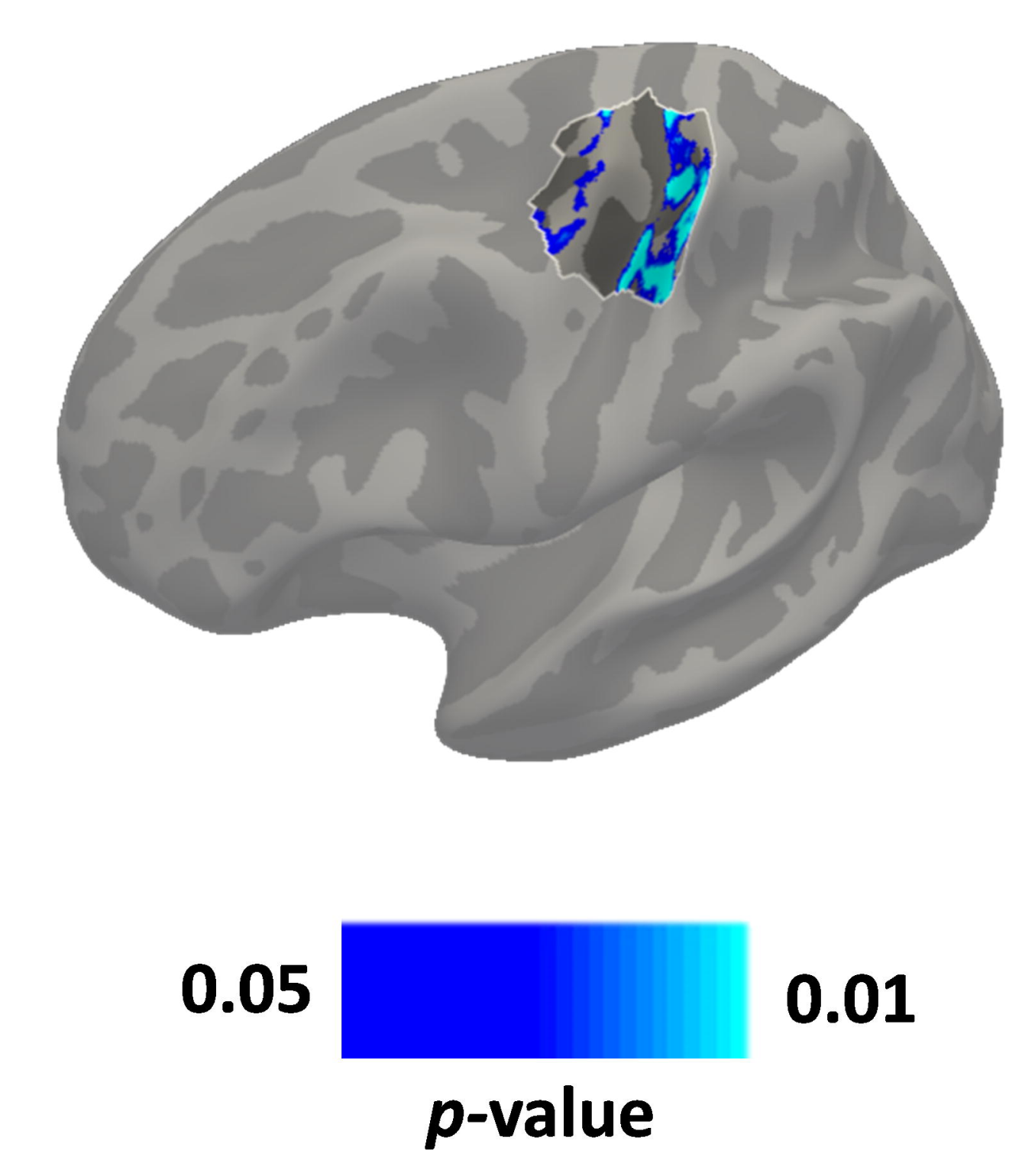

B
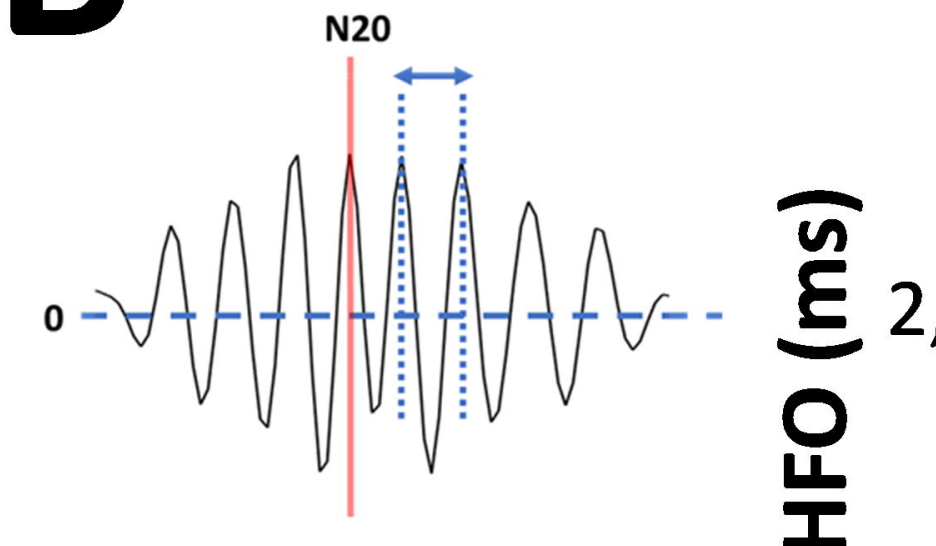

हู 2,2

\section{correction analysis \\ Cluster-based}

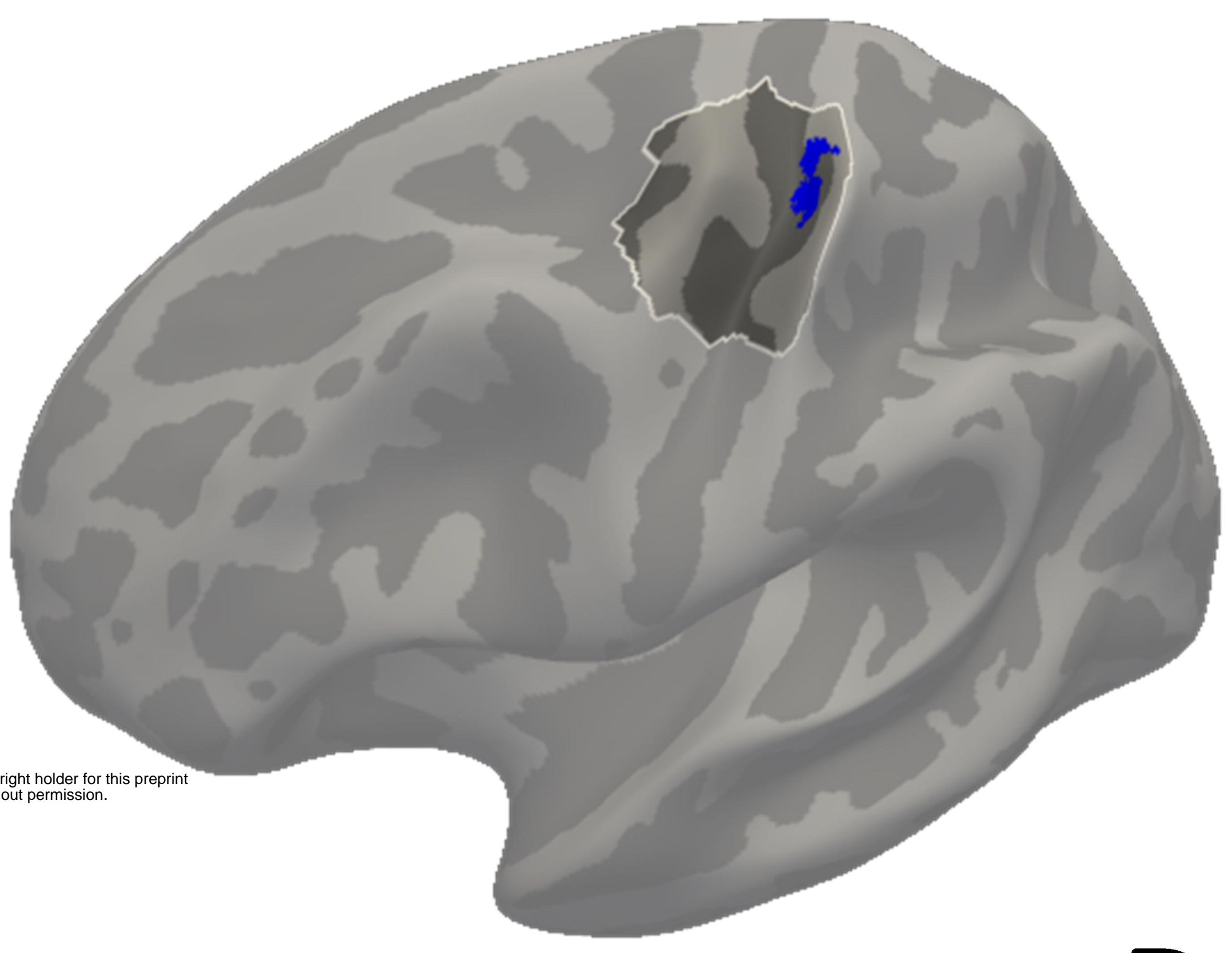

D

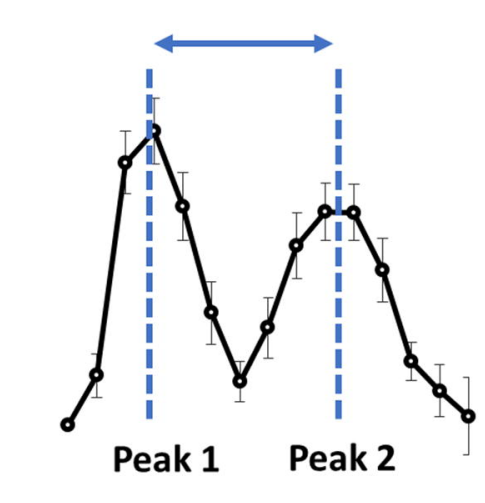

Cluster-based correction analysis

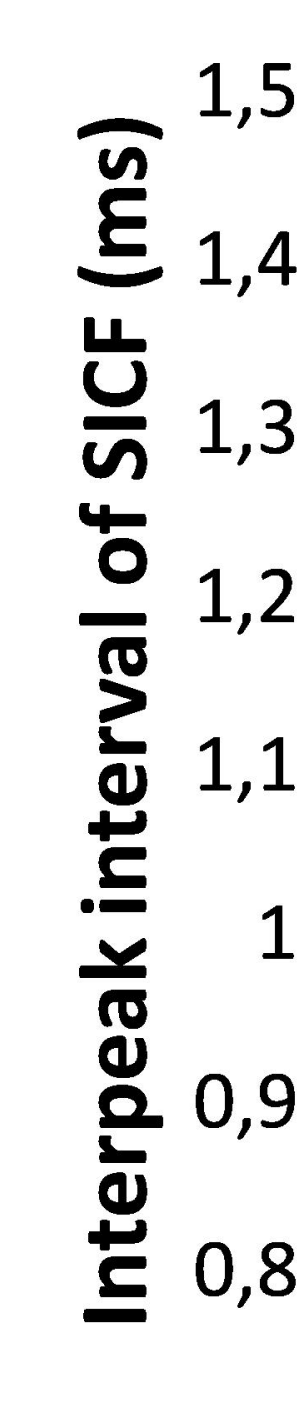

Cortical Myelination and late s-HFO frequency

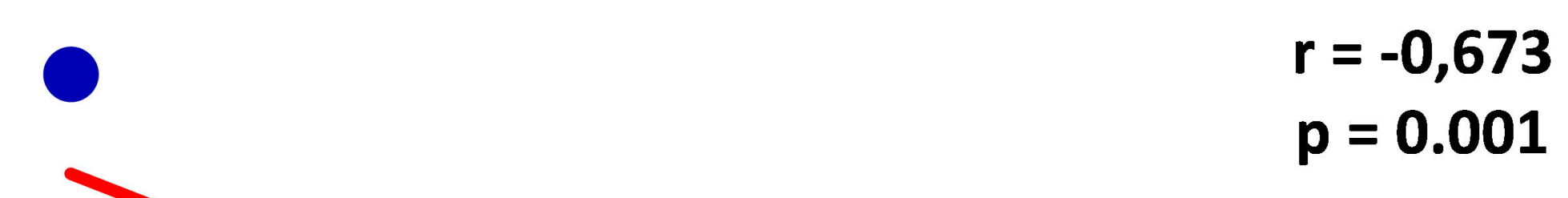

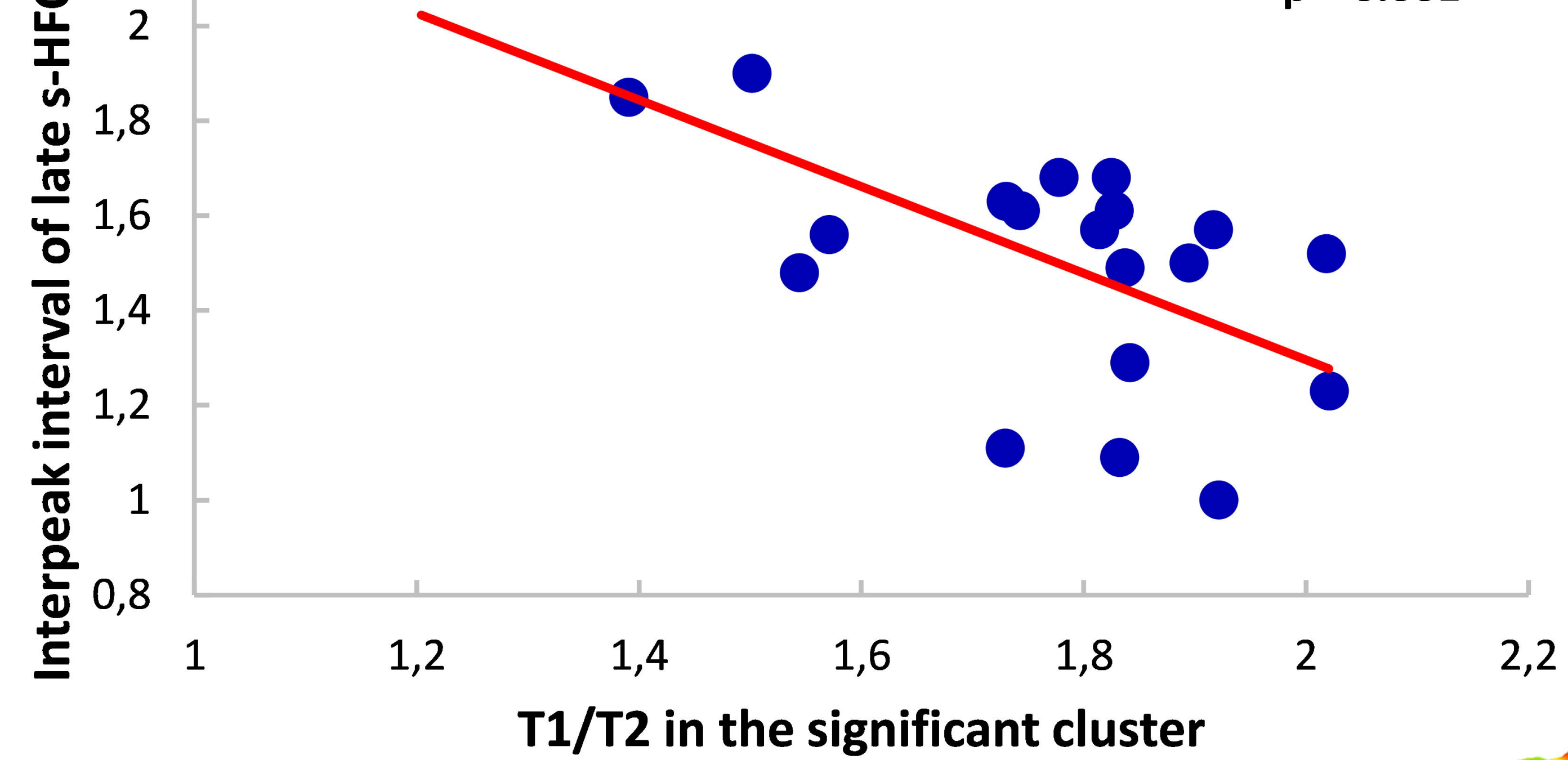

$\mathrm{T} 1 / \mathrm{T} 2$ in the significant cluster

Cortical Myelination and SICF frequency



